Check for updates

Cite this: RSC Adv., 2017, 7, 43161

Received 14th July 2017

Accepted 16th August 2017

DOI: $10.1039 / \mathrm{c} 7 \mathrm{ra07719j}$

rsc.li/rsc-advances

\title{
Potentiation effect of HB-EGF on facilitating wound healing via 2-N,6-O-sulfated chitosan nanoparticles modified PLGA scaffold
}

\author{
Xiang Peng, (D) ab Yuanman Yu, ${ }^{\text {ab }}$ Zihao Wang, ${ }^{\text {ab }}$ Xiaohui Zhang, ${ }^{b}$ Jing Wang ${ }^{\star a b}$ \\ and Changsheng Liu (D)*ab
}

\begin{abstract}
Heparin-binding epidermal growth factor (HB-EGF) is of extreme importance for accelerating wound healing; however, its effective and controlled release remains to be solved. In this study, 2-N,6-Osulfated chitosan (26SCS), a highly sulfated polysaccharide, was utilized to fabricate delivery vehicles of HB-EGF encapsulated in PLGA nanofibers to achieve effective release. In addition, migration of Hacat cells was greatly promoted by 26SCS synergizing with HB-EGF released from the PLGA scaffold and proliferation was not affected. In a mouse excisional splinted model, a wound treated with HB-EGF loaded S-PLGA nanofibers was basically healed at 14 days. Conversely, wounds in other groups, including HB-EGF-loaded PLGA nanofibers, were not closured. The acceleration of wound healing was ascribed to the 26SCS-favored HB-EGF effect on the migration of keratinocyte which led to rapid reepithelialization. Furthermore, neovascularization was also observed in the HB-EGF loaded S-PLGA nanofiber treated wound. These data strongly proved that S-PLGA nanofibers with incorporated HB-EGF may provide a promising way for rapid regeneration of the cutaneous wounds.
\end{abstract}

\section{Introduction}

Skin wounds resulting from burns, tumors, or excision remain as tremendous challenges. Cutaneous wounds affect up to 100 million patients every year. ${ }^{1}$ Although dressing remains the standard of wound care, insufficient availability of dressing complicates wound closure and increases the risk of infection in patients with large sized wounds. Current tissue engineered skin substitutes have become increasingly more accepted with their ability to favor skin restoration. However, they have limited effects on the formation of functional tissue. ${ }^{2}$ In addition, accelerating healing is also a goal in therapeutic treatment that avoids converting an acute wound into a chronic wound. The ideal skin graft involves several strategies such as biodegraded scaffold with analogous extracellular matrix (ECM) structures, ${ }^{3}$ the use of functional cellular components, and growth factors that might synergistically enable regrowth of a functional and physiological dermal layer of skin. ${ }^{4}$

Regeneration of tissue after skin injury is initiated via several growth factors involving a fibroblast growth factor (FGF) family, platelet-derived growth factor (PDGF) family, epidermal growth factor (EGF) family, etc. The latter is comprised of EGF, heparin-

${ }^{a}$ Key Laboratory for Ultrafine Materials of Ministry of Education, East China University of Science and Technology, Shanghai 200237, PR China.E-mail: biomatwj@163.com; Tel: +86-021-64251308

${ }^{b}$ Engineering Research Center for Biomedical Materials of Ministry of Education, East China University of Science and Technology, Shanghai 200237, PR China binding EGF (HB-EGF), and trans-forming growth factor- $\alpha$ (TGFa). ${ }^{5}$ HB-EGF was identified as the major heparin-binding growth factor in wound fluid of porcine partial-thickness excisional wounds. ${ }^{6}$ As a versatile growth factor participating in skin regeneration, HB-EGF functions by accelerating keratinocyte migration, rather than proliferation during wound healing. ${ }^{7}$ Furthermore, HB-EGF is mitogenic for fibroblasts and can synergize with Insulin-like Growth factor-I (IGF-I). ${ }^{6,8}$ Interestingly, HB-EGF also plays an important role in reepithelialization and granulation tissue formation.

Although application of exogenous growth factors can accelerate wound healing, they have difficulty in maintaining bioactivities over a rather long duration due to the short in vivo half-lives of most growth factors. It seems likely that a delivery system that protects growth factors from rapid degradation and denaturation would be more effective to facilitate wound healing. For example, EGF-conjugated nanofiber through functional amine groups could potentially be employed as a novel wound healing material. ${ }^{9}$ Recently, heparin-containing delivery systems have been used to immobilize high affinity heparinbinding growth factors and overcome a burst release. ${ }^{\mathbf{1 0 , 1 1}}$ Besides, sulfated or sulfonated materials that might mimic this biochemical function of an ECM have been proposed for designing a delivery system. Specifically, our group has demonstrated that 2- $\mathrm{N}, 6-\mathrm{O}$-sulfated chitosan (26SCS), a highly sulfated heparin-like polysaccharide, not only can control release of BMP-2 and VEGF, but also has a synergistic effect with these heparin-binding growth factors. ${ }^{12,13}$ However, up to now, 
few studies have been performed on this heparin-like sulfated chitosan system as a versatile carrier to deliver HB-EGF. Furthermore, feasible interactions between 26SCS and HBEGF have yet to be investigated.

In view of this, we placed an emphasis on the effects of 26SCS with HB-EGF on the migration and proliferation of keratinocytes in vitro, as well as the fabrication of 26SCSembedded poly(lactic-co-glycolic acid) (PLGA) nanofibrous matrix (S-PLGA) for HB-EGF loading, aiming at full-thickness skin regeneration in this research. Our strategy to immobilize HB-EGF takes advantage of the affinity between 26SCS and HB-EGF. Investigations including morphological observation, plus loading and releasing profiles were carried out, as well as bioactivity of the released HB-EGF. Furthermore, we assessed the effect of HB-EGF loaded S-PLGA scaffolds on wound healing in a mouse excisional splinting model by optical images, histological evaluation, immunohistochemistry, and immunofluorescence analysis. We hypothesized that the heparin-like 26SCS may cooperate with HB-EGF not only for binding and controlled release, but also may have a synergistic effect on promoting wound healing.

\section{Materials and methods}

\section{Materials}

Poly(lactic-co-glycolic acid) with a $50: 50$ monomer ratio and average molecular weight of $100 \mathrm{kDa}$ was purchased from Jinan Dai Gang Biological Technology Co., Ltd. (Jinan, China). Toluidine blue zinc chloride double salt, 3-(4,5dimethylthiazol-2-yl)-5-(3-carboxymethoxyphenyl)-2-(4-sulfophenyl)- $2 H$-tetrazolium (MTS) was purchased from SigmaAldrich (St. Louis, MO, USA), recombinant human HB-EGF was obtained from PeproTech (Rocky Hill, NJ, USA). All cellculture-related reagents were available from Gibco (Grand Island, NY, USA).

\section{Cell culture}

Hacat cells were purchased from American Type Culture Collection (ATCC, VA, USA) and grown in Dulbecco's modified Eagle's medium (DMEM) supplemented with 10\% fetal bovine serum (FBS), $100 \mathrm{U} \mathrm{mL}^{-1}$ penicillin, $100 \mathrm{mg} \mathrm{mL}^{-1}$ streptomycin (cell culture reagents were obtained from Sigma-Aldrich, Saint Louis, MO, USA).

\section{Fabrication of S-nanoparticles}

2- $N, 6-O-S u l f a t e d$ chitosan (26SCS), synthesized as previously described, was used to fabricate modified-nanoparticles (S-NP). ${ }^{14}$ Briefly, in this experiment, chitosan $\left(M_{\mathrm{w}}=3 \times 10^{5}\right)$ was dissolved in $2 \%$ acetic acid, forming a $0.02 \%(\mathrm{w} / \mathrm{v})$ positively-charged chitosan solution, which interacts with negatively-charged 26SCS solution that was formed by 26SCS $\left(10 \mathrm{mg} \mathrm{mL}^{-1}\right)$ in $50 \mathrm{mM}$ sodium sulfate (SS) through polyvalent charge attraction; this formed a highly-sulfated glycosaminoglycan-containing coacervate.

\section{Electrospinning of nanofibers}

The electrospinning apparatus consisted of a $10 \mathrm{~mL}$ syringe (Popper \& Sons, Inc., New Hyde Park, NY, USA) connected to a syringe pump (Orian Sage, Fisher, Fair Lawn, NJ, USA). A highvoltage power supply (Glassman Series EH, High Bridge, NJ, USA) was used to apply a voltage to the needle. The collector consisted of a $5^{\prime \prime} \times 5^{\prime \prime}$ metal sheet covered in non-stick aluminum foil, which was placed $18 \mathrm{~cm}$ from the tip of the needle to collect the dried fibers. PLGA was dissolved in a solvent of 1,1,1,3,3,3-hexafluoro-2-propanol (HFIP) to achieve a concentration of $17 \mathrm{wt} \%$ and certain amounts of S-NP (the weight percent of S-NP was $10 \%$ with respect to PLGA used) were added to the solution. The solution involving PLGA and S-NP was stirred for $12 \mathrm{~h}$ at room temperature. The condition was optimized from evaluations of stability and homogeneity of the solutions. The S-PLGA nanofibers were electrospun using a needle $(0.51 \mathrm{~mm}$ inner diameter $)$, under a condition of $1.2 \mathrm{~mL} \mathrm{~h}{ }^{-1}$ flow rate, $+11 \mathrm{kV}$ voltage, and $18 \mathrm{~cm}$ working distance. Meanwhile, pure PLGA nanofibers were also fabricated under the same conditions to serve as a control. The aluminum foil covered with nanofibers was dried overnight under vacuum and used for further studies.

\section{Morphological investigation}

The particle sizes and morphology of S-NP were examined by transmission electron microscopy (TEM). The morphology of the electrospun nanofibers containing S-NP or no S-NP was studied using scanning electron microscopy (SEM; S-3400, Hitachi, Tokyo, Japan) at an accelerating voltage of $15 \mathrm{kV}$, after sputter coating with gold.

\section{Toluidine blue staining assay}

The S-NP on nanofibers was determined via a toluidine blue stain assay. S-PLGA nanofibers and PLGA nanofibers were incubated for $1 \mathrm{~h}$ in a toluidine solution (aqueous solution containing $0.005 \%(\mathrm{w} / \mathrm{v})$ toluidine blue zinc chloride double salt, $0.01 \mathrm{M}$ hydrochloric acid, and $0.2 \%(\mathrm{w} / \mathrm{v})$ sodium chloride) at room temperature. A purple hue resulted on the surface of the S-PLGA nanofibers, while PLGA nanofibers remained white.

\section{HB-EGF loading efficiency}

The loading efficiency of HB-EGF was determined by a Human HB-EGF ELISA kit. Briefly, modified PLGA nanofibers and SPLGA nanofibers $\left(64 \mathrm{~mm}^{2}\right)$ were placed in a 48 -well plate with $500 \mu \mathrm{L}$ of phosphate-buffered saline (PBS) containing $1 \mu \mathrm{g} \mathrm{HB}$ EGF, incubated at $37{ }^{\circ} \mathrm{C}$ under continuous agitation and left $24 \mathrm{~h}$ to allow protein adsorption. Then $250 \mu \mathrm{L}$ of solution was collected and stored frozen, and later examined with the released HB-EGF using an ELISA kit according to the kit instructions.

\section{HB-EGF release profile}

Released HB-EGF from nanofibers was also determined using a Human HB-EGF ELSIA kit. Briefly, modified nanofibers were placed in a 48 -well plate. $500 \mu \mathrm{L}$ of PBS solution containing 
$1 \mu \mathrm{g}$ HB-EGF was added to the plate and left $24 \mathrm{~h}$ to be absorbed. Then the HB-EGF loaded nanofibers were lyophilized and $500 \mu \mathrm{L}$ of PBS was transferred to the plate that was covered by the HB-EGF loaded nanofibers, and incubated at $37^{\circ} \mathrm{C}$ under continuous agitation. At regular intervals, $10 \mu \mathrm{L}$ of solution was collected and stored frozen, and an equal amount of fresh PBS was added to the released medium. In the end, all collected samples were thawed and measured by a Human HB-EGF ELISIA kit. All samples were studied in triplicate.

\section{Scratch wound assay}

A 48 well plate was seeded with the immortal human keratinocyte line Hacat cells to a final density of $5 \times 10^{4}$ per well and these were maintained in Dulbecco's modified Eagle's medium (DMEM) with $2 \%$ fetal bovine serum (FBS) at $37{ }^{\circ} \mathrm{C}$ for $24 \mathrm{~h}$ to permit cell adhesion and the formation of a confluent monolayer. Furthermore, 2\% FBS was used in the culture medium to suppress cell proliferation for the sake of limiting that influence on wound closure. A $200 \mu \mathrm{L}$ pipette tip was used to scratch these confluent monolayers and then they were washed twice with PBS to remove dislodged cells. Cells were incubated with DMEM with $2 \%$ FBS at $37{ }^{\circ} \mathrm{C}$, containing $80 \mathrm{ng} \mathrm{mL}{ }^{-1} \mathrm{HB}$-EGF loaded S-PLGA nanofibers or free HB-EGF, or no HB-EGF as control. Wound closure in vitro was monitored by collecting digitized images at $7 \mathrm{~h}$ intervals after performing a scratch until the wound had almost healed. Digitized images were captured with an inverted microscope and then analyzed using Image-J software to measure the scratch width. All scratch assays were performed in triplicate.

\section{Cell proliferation assay}

The immortal human keratinocyte line Hacat cells were seeded at a density of $1 \times 10^{4}$ cells per $\mathrm{mL}$ in a 48 -well plate and cultured in Dulbecco's modified Eagle's medium (DMEM) with $10 \%$ fetal bovine serum (FBS) at $37{ }^{\circ} \mathrm{C}$, containing either $10 \mathrm{ng} \mathrm{mL}{ }^{-1}$ HB-EGF loaded S-PLGA nanofibers or free HB-EGF, or no HB-EGF as control. A colorimetric MTS assay was used to determine viable cells on the substrates after 3 days of culturing. All samples were tested in triplicate.

\section{Wound model and analysis}

20 male C57BL6/J mice (6 weeks old, Slac, Inc. Shanghai, China) were divided into four groups and individually anesthetized using an intraperitoneal injection of isoflurane and the dorsal surface of each was shaved. The dorsa of the mice were rinsed with an iodophor swab and then a $5 \mathrm{~mm}$ punch biopsy tool was used to make identical full-thickness wounds on the dorsa of the mice. Two wounds were made on each side of the dorsal midline. Two $0.5 \mathrm{~mm}$-thick silicone donut-shaped splints $(\mathrm{OD}=$ $15 \mathrm{~mm}, \mathrm{ID}=9 \mathrm{~mm}$ ) were placed so that the wound was centered within the splint. A 5-0 Prolene suture was used to fix the splint to the skin after using an immediate-bonding adhesive. There were four groups: PLGA nanofibers (PLGA), $1 \mu \mathrm{g}$ HB-EGF-loaded PLGA nanofibers (PLGA + HB-EGF), S-PLGA nanofibers (S-PLGA), or $1 \mu \mathrm{g}$ HB-EGF-loaded S-PLGA nanofibers (S-PLGA + HB-EGF), which were applied to the wounds, respectively ( $n=5$ mice/10 wounds per group). These nanofibers were tailored to the same diameter of that of the wound. To prevent chewing of the splints, occlusive dressing (Tegaderm, 3M) was placed to cover each dorsal surface. General observations were carried out using digital photographs on days $0,7,10$, and 14 . Wound area was analyzed by tracing the wound margin with a fineresolution computer mouse and calculating pixel area using Image-J software. On day 7, two mice per group were euthanized and wounds were excised, bisected, and fixed in $10 \%$ paraformaldehyde for histological evaluation. On day 14, remaining mice were euthanized and treated as described above. All procedures were performed in strict accordance with the NIH guidelines for the care and use of laboratory animals (NIH Publication no. 85-23 Rev. 1985) and were approved by the Research Center for Laboratory Animal of Shanghai University of Traditional Chinese Medicine.

\section{Histological analysis}

The skin sections underwent routine histological processing with hematoxylin and eosin (HE) for standard evaluation and with Masson's trichrome staining (MTS) to observe collagen content. Dyed sections were photographed using an inverted microscope. All samples for histological analysis were performed on more than 4 wounds per group at each time point. Images presented are representative of all replicates.

\section{Immunohistochemical and immunofluorescent analysis}

Immunohistochemical staining was used to determine the production of cytokeratin as a marker of epithelial cells during wound healing. Fixed tissue sections were treated with a rabbit polyclonal anti-wide spectrum cytokeratin antibody $(1: 150$, Abcam), using an IHC staining kit (Sigma), then counterstained with hematoxylin, and examined by inverted microscopy. Frozen sections on Day 7 were dual stained with rabbit polyclonal antiki-67 (1:100, Abcam) and mouse monoclonal anti-integrin $\beta 4$ (1 : 100, Abcam), followed by goat anti-rabbit IgG Alexa Fluor 594 ( $1: 200$, Abcam) and goat anti-mouse IgG FITC secondary antibodies (1:200, Abcam), counterstained with DAPI. Day 14 frozen sections were dual-stained with CD-31 (1:100, Abcam) and anti- $\alpha$-smooth actin $(1: 200$, Abcam), then treated with goat anti-rabbit IgG Alexa Fluor 594 (1:200, Abcam) and goat antimouse IgG FITC secondary antibodies (1:200, Abcam), counterstained with DAPI. All frozen sections were examined by confocal laser scanning microscopy (Nikon, Japan).

\section{Statistical analysis}

All quantitative data expressed as the mean \pm SD were analyzed with Origin 9.0 (OriginLab Corp, USA). Statistical differences were detected using one-way analysis of variance (ANOVA). Statistical significance was accepted at $p<0.05$.

\section{Results}

\section{Morphology of nanofibers and S-nanoparticles}

Morphological images of the S-NP loaded and the neat PLGA nanofibers were taken using scanning electron microscopy (SEM) and are shown in Fig. 1A and B, respectively. The SEM 
images depict randomly interconnected structures and the seemingly smooth morphology of the PLGA nanofibers and SPLGA nanofibers. Besides, the inserted images of S-PLGA nanofibers stained with toluidine blue (TB), in contrast to the stained PLGA nanofibers, became purple, which demonstrates that the nanoparticles were evenly distributed among the nanofibers. ${ }^{15}$ The diameter is about $1.93 \mu \mathrm{m}$ for the S-PLGA nanofibers and about $1.74 \mu \mathrm{m}$ for the PLGA nanofibers, as determined by using image analysis software. Fig. 1C shows a transmission electron microscopy (TEM) image of the S-NP, with the diameter of these S-NP being $109 \mathrm{~nm}$.
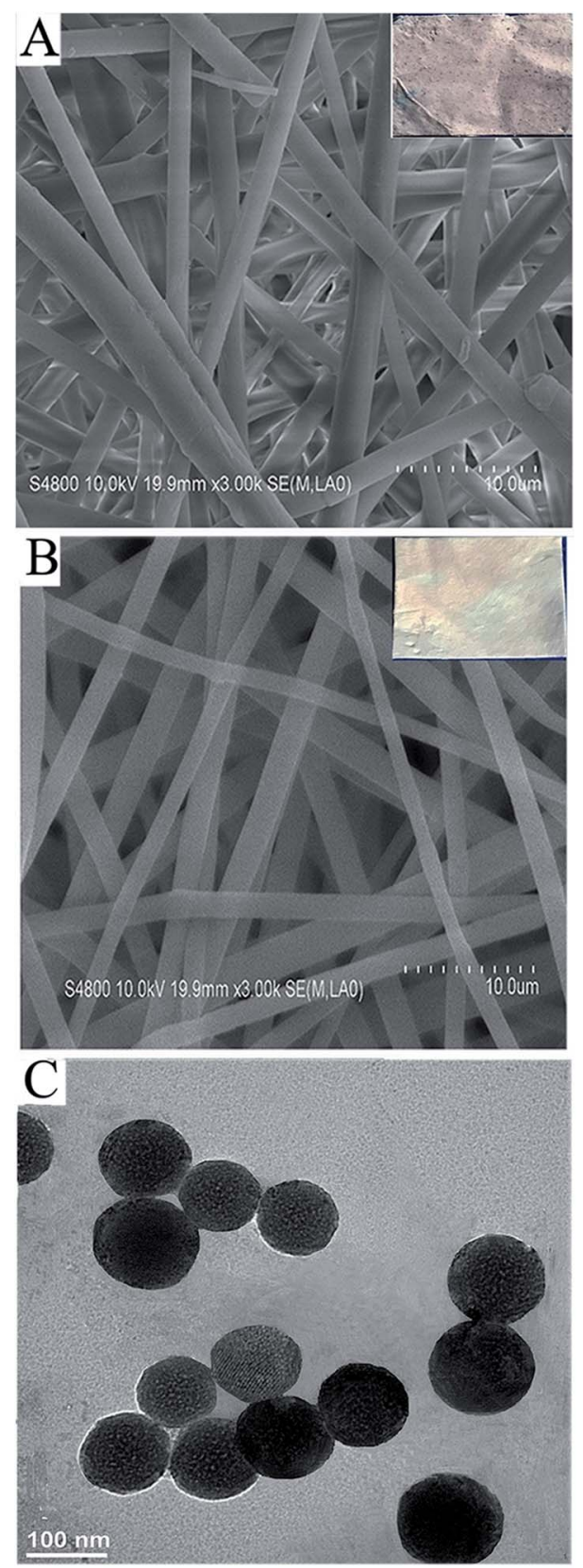

Fig. 1 SEM images of nanofibers: (A) S-PLGA nanofibers; the insert image is that of nanofibers stained with toluidine blue, which proves SNP is well-distributed in PLGA nanofibers. (B) PLGA nanofibers. (C) TEM images of S-NP.

\section{Loading efficiency and release kinetics of HB-EGF}

The loading efficiencies of HB-EGF for the neat and S-NP-loaded PLGA nanofibers were determined by ELISA kits. As depicted in Fig. 2A, the HB-EGF loading efficiency of S-PLGA nanofibers was 1.85 fold higher than that of PLGA nanofibers. The binding affinity between S-NP and HB-EGF might contribute to enhancing the loading capacity of HB-EGF. To investigate the release behavior of HB-EGF from both delivery vehicles, the release profiles of HB-EGF were determined and are presented in Fig. 2B. HB-EGF released from PLGA nanofibers showed an initial burst of approximately $37 \%$ after $24 \mathrm{~h}$, while that of SPLGA was about $17 \%$. Thereafter, the release was almost linear and sustained through the 10 day experiment. At 10 days, a cumulative release was observed of $56 \%$ from S-PLGA nanofibers compared with $83 \%$ for the PLGA nanofibers, indicating the alleviated bolus and sustained release.

\section{Bioactivity of HB-EGF}

Cell migration in vitro. The effect of free HB-EGF and HBEGF-loaded S-PLGA nanofibers on the migration of Hacat cells was evaluated by a wound scratch assay. As revealed in Fig. 3A, there are substantial statistics showing the effect of free HB-EGF
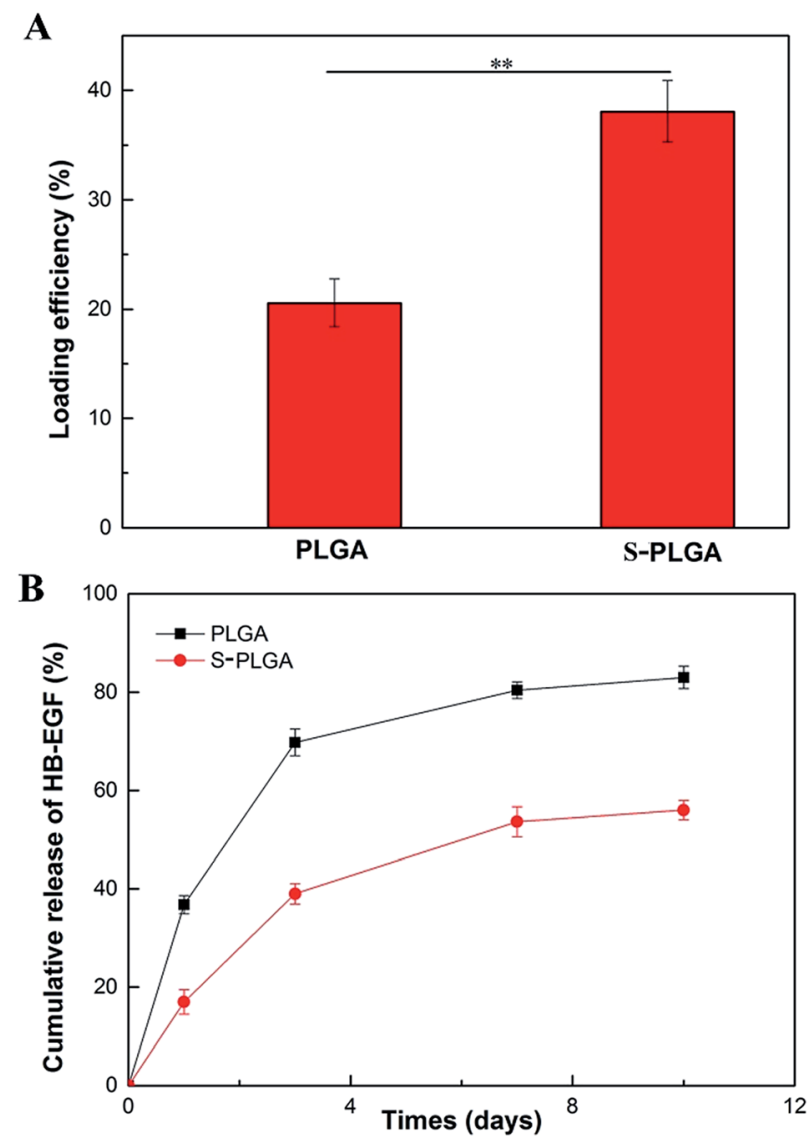

Fig. 2 (A) Binding efficiency of HB-EGF to PLGA and S-PLGA nanofibers. The values represent the mean \pm standard deviation $(n=3), * p<$ $0.05, * * p<0.01$. (B) Release kinetics of HB-EGF from PLGA and SPLGA nanofibers. 
A

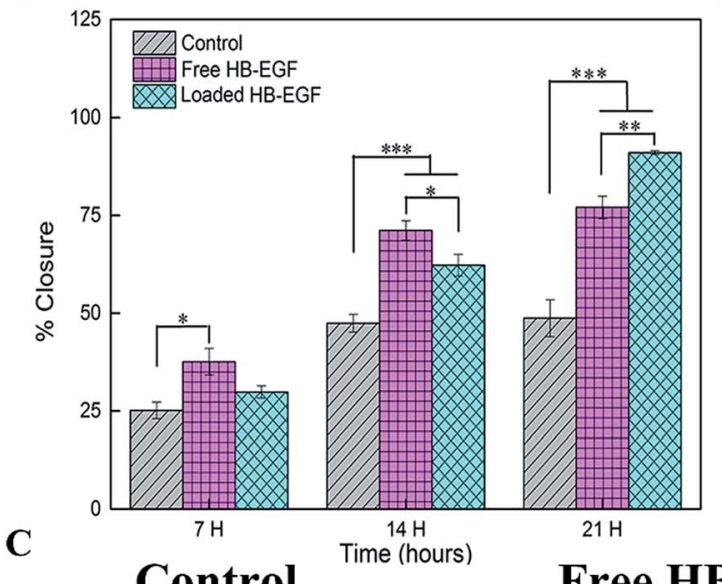

B

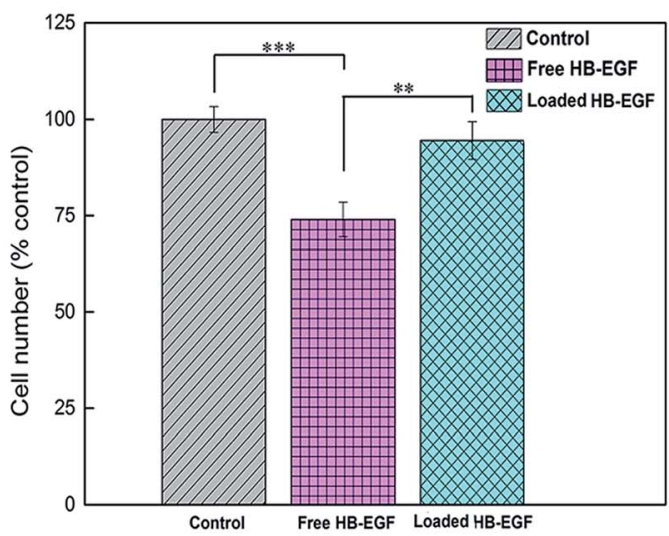

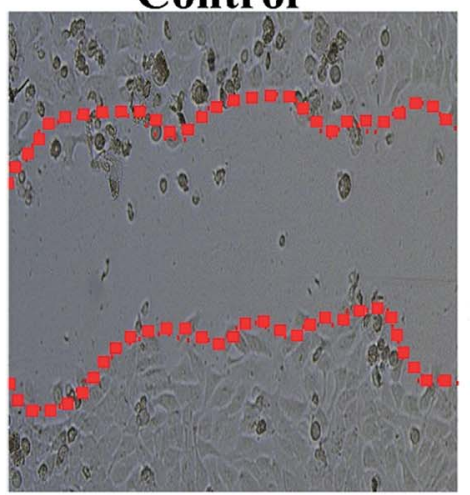

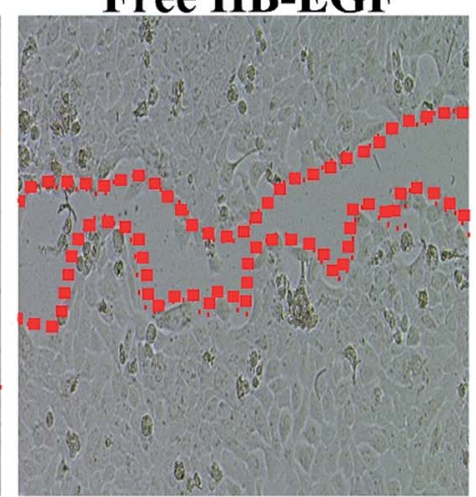

Loaded HB-EGF

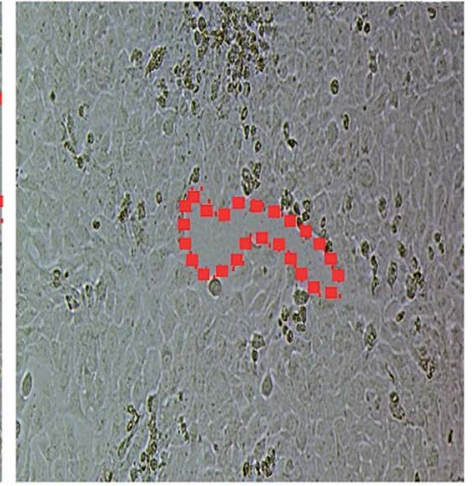

Fig. 3 HB-EGF loaded in the S-PLGA promoted the migration of Hacat cells, while not inhibiting proliferation in vitro. (A) Quantification of percent closure of scratch wounds on confluent Hacat cell monolayers over $21 \mathrm{~h}$ in low-serum (2\% FBS) medium added without HB-EGF as control, $80 \mathrm{ng} \mathrm{mL}^{-1}$ free HB-EGF or $80 \mathrm{ng} \mathrm{mL}^{-1} \mathrm{HB}-\mathrm{EGF}$ loaded in S-PLGA nanofiber. The values represent the mean \pm standard deviation $(n=$ 3), $* p<0.05, * * p<0.01, * * * p<0.001$. (B) Quantification of cell numbers after 3 days, Hacat cells cultured in culture medium as control, supplemented with $10 \mathrm{ng} \mathrm{mL}^{-1}$ free HB-EGF or $10 \mathrm{ng} \mathrm{mL}^{-1} \mathrm{HB}-\mathrm{EGF}$-loaded in S-PLGA nanofibers, ** $p<0.01, * * * p<0.001$. (C) Representative images of scratch wounds.

on improving wound closure compared with the control at 7 hours after wounding, while there is no significant distinction between the free HB-EGF and the HB-EGF loaded samples. At the time point of 14 hours, the free HB-EGF and HB-EGF loaded samples both greatly accelerated the rate of wound healing compared with the control, and free HB-EGF had a slightly greater advantage over the HB-EGF loaded one for wound closure. Nevertheless, by 21 hours post-wounding, the HB-EGF loaded sample had more contributions speeding up the process of wound closure than the free HB-EGF one. The course of the closuring wound was tracked and a representative image over $21 \mathrm{~h}$ is shown in Fig. 3C. It is obvious that the wound in the HB-EGF loaded group had almost closured in contrast to the free HB-EGF group. In fact, although the same dose of HB-EGF was added to both groups ( $80 \mathrm{ng} \mathrm{mL}^{-1}$ ), the loaded group had a fairly lower HB-EGF concentration in the culture medium, especially at an early stage of release. Therefore, the S-NP not only serves in a role for controlled release of the HB-EGF, but also has a synergistic effect on the migration of Hacat cells with HB-EGF.

Cell proliferation in vitro. Hacat cells proliferation on free HB-EGF and HB-EGF-loaded S-PLGA nanofibers with an equal dose of growth factor was studied at 3 days, with results shown in Fig. 3B. It was observed that free HB-EGF greatly suppressed cell growth compared with the control and showed a significant statistical difference, while no statistically significant difference in cell proliferation between the HB-EGF loaded and the control group was observed. Furthermore, statistical discrepancy in cell proliferation existed between the HB-EGF loaded and the free HB-EGF group. Notably, inhibition of cell proliferation in the HB-EGF loaded group was substantially weaker than that of the free HB-EGF one. This is consistent with the premise of controlled release reducing the burst effect.

Murine wound analysis. The effect of HB-EGF loaded on wound closure was determined by measuring the area of a wound over 14 days in a splinted mouse full-thickness excisional wound model. The advantage of this model is that skin is attached to the underlying cartilage, splinting the wound, which impedes wound contraction and allows gradual wound healing to occur by re-epithelialization and granulation tissue formation. As displayed in Fig. 4B, at 7 days, the HB-EGF-loaded SPLGA nanofibers (HB-EGF + S-PLGA) group had vast advantages of quick wound healing compared with other groups and kept it throughout the trial. A dramatic decrease in the wound 
A

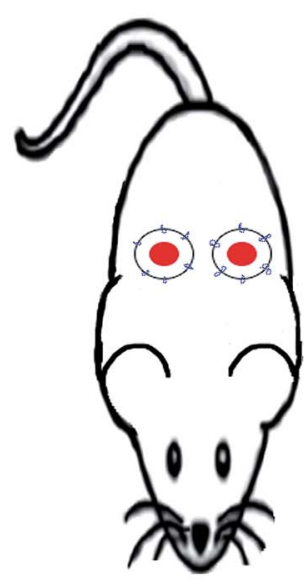

B

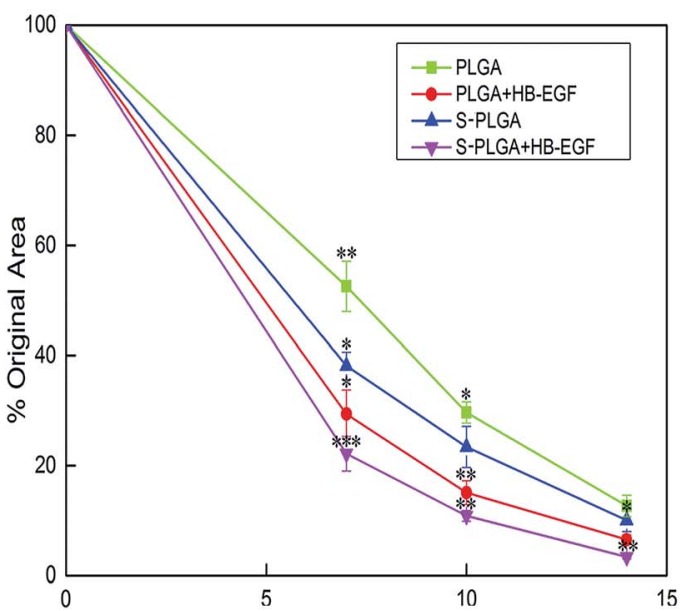

C

C PLGA
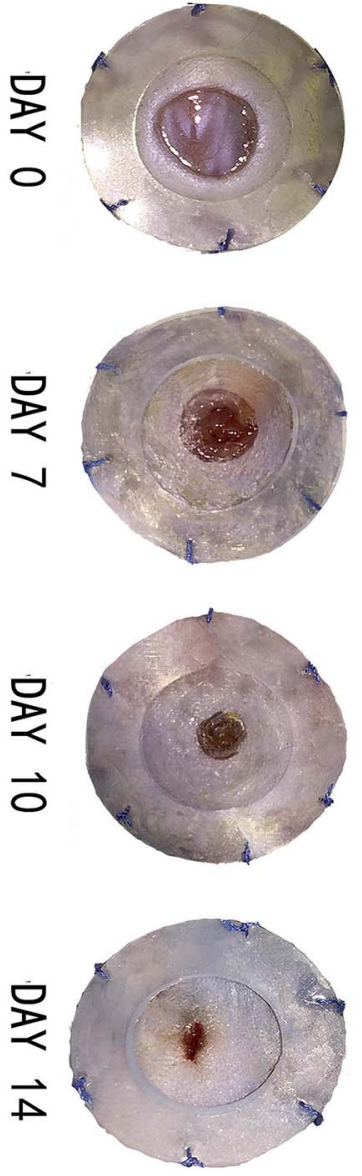

PLGA+HB-EGF
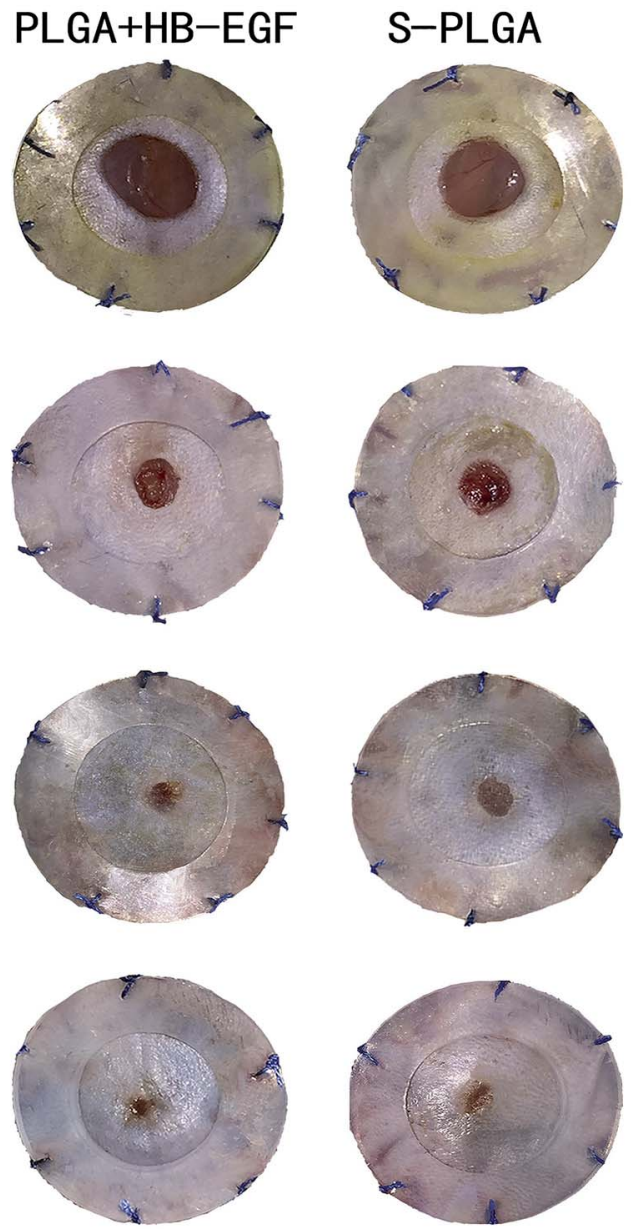
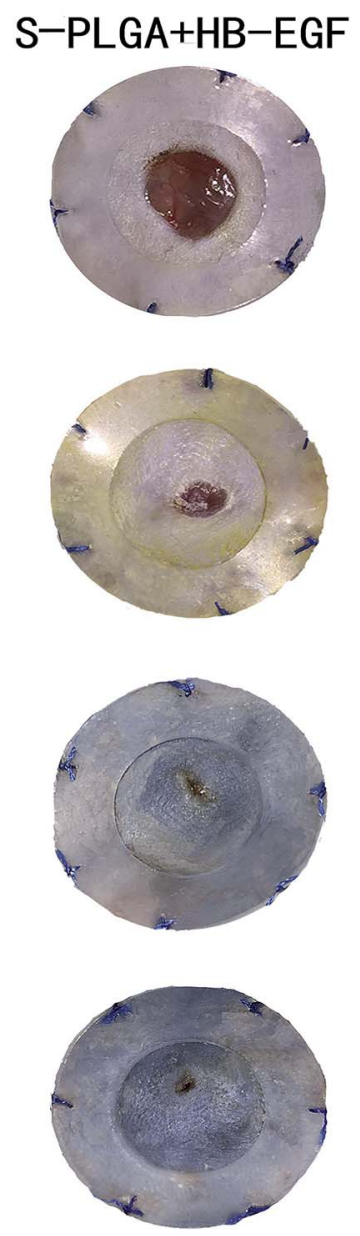

Fig. 4 The HB-EGF loaded S-PLGA nanofibers accelerates wounds closure. (A) Secured silicone splint to the skin with six sutures surrounding the wounds. Wounds were treated immediately with the PLGA nanofibers; $1 \mu \mathrm{g} \mathrm{HB}$-EGF + PLGA nanofibers, the S-PLGA nanofibers and $1 \mu \mathrm{g}$ HBEGF + S-PLGA nanofibers, respectively. (B) Time-dependent changes in the wound area determined with image analysis software (area of actual wound/area of original wound $\times 100$ ), $* p<0.05, * * p<0.01, * * * p<0.001$. (C) Representative images of wounds treated with four groups were tracked at 7 days, 10 days, and 14 days.

area was observed in the case of the S-PLGA + HB-EGF group with $77 \%$ healing occurring in 7 days. At the same time interval, wounds treated with other groups healed no more than $70 \%$. At 10 days, wounds in the S-PLGA + HB-EGF group healed about
86\%. This still held an advantage for speeding up wound healing compared with other groups. Furthermore, epithelialization appeared to have nearly completed by 14 days and there was a statistically significant difference in the wound area 
between every time point. However, the wounds were not completely closured in other groups.

Histological examination was conducted on wound tissue to analyze the re-epithelialization by $\mathrm{H} \& \mathrm{E}$ and the formation of collagen by Masson's trichrome staining (MTS).${ }^{16}$ Images of the stained sections after 7 days are presented in Fig. 5A. A thick epidermal layer and granulation region was noticed in the case of S-PLGA + HB-EGF, indicating accelerated re-epithelialization of the HB-EGF-loaded S-PLGA nanofibers. In addition, the SPLGA + HB-EGF group exhibited an organized collagen deposition which might favor formation of granulation tissue, while other groups contained much less collagen and showed a thin or inconsistent granulation regions. As depicted in Fig. 5B, immunohistochemical exploration of cytokeratin, the S-PLGA +
HB-EGF group revealed a thick stratum corneum in 7 days. What's more, new dermal appendages formed in the wounded region that were identical to the surrounding normal epidermis in 14 days. However, the newly forming dermal appendages did not appear in other groups. ${ }^{8}$

Cell viability in vivo. The effect of HB-EGF on the proliferation of keratinocytes was assessed at 7 days post-wounding by using Ki-67 (a marker of cell proliferation) immunofluorescence. Meanwhile, the migration of keratinocytes was determined by stained $\beta 4$ integrin that acts to activate Rac1 for coordinated cell motility during wound healing. The PLGA group showed a double-negative throughout the wound region. The HB-EGF + PLGA group induced the migration of nonproliferating (Ki-67-negative) cell, which was consistent with
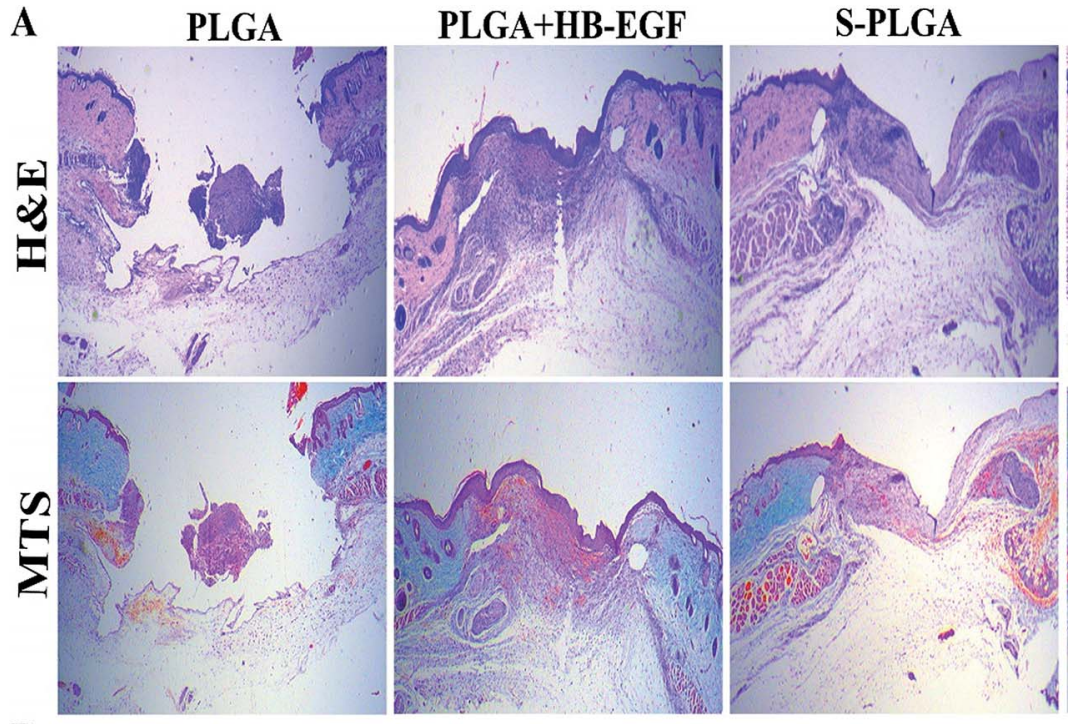

\section{S-PLGA+HB-EGF}

B

PLGA
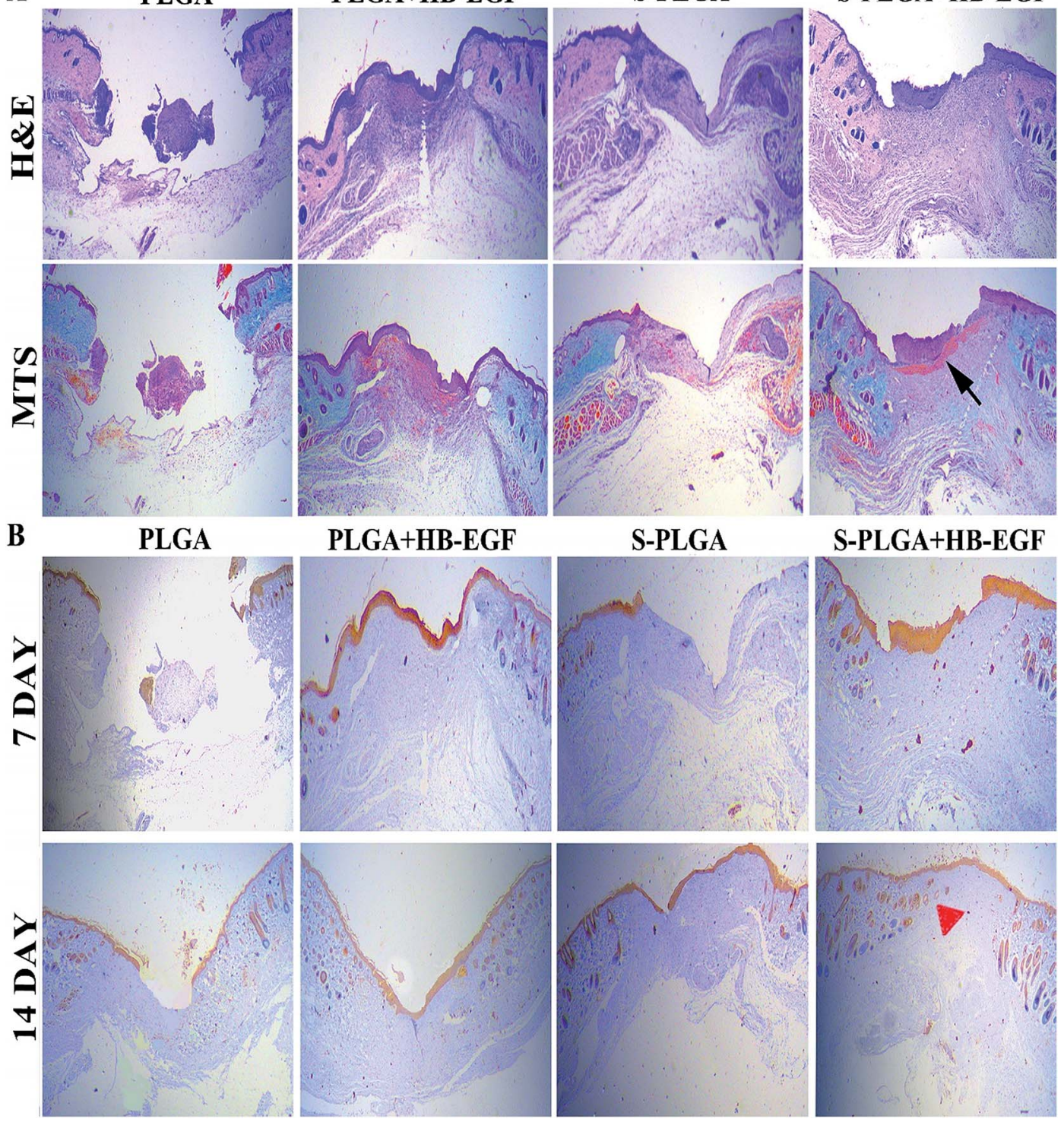

Fig. 5 Histological analysis of wound region. (A) Representative images of wound sections at 7 days after injury stained with hematoxylin and eosin (H\&E) for observing the re-epithelialization and Masson's trichrome staining (MTS) for examination of collagen content that indicates granulation tissue. Black arrows indicate continuous collagen deposition. (B) Immunohistochemical detection for cytokeratin, which is a marker of epithelial wounds. Red arrows indicate newly formed dermal appendages. 
the result of suppressed cell proliferation in vitro. Notably, there were several cells indicating proliferation (Ki-67-positive) and fewer cell migrations ( $\beta 4$ integrin-negative) presented in the $S$ PLGA group, which suggested that 26SCS modified PLGA nanofibers induced the proliferation of keratinocyte. Wounds in the HB-EGF + S-PLGA group exhibited many more proliferating cells compared with those in other groups and the migration of proliferating cells was observed (double-positive), as shown in Fig. 6A, indicating that the HB-EGF-loaded SPLGA nanofibers promoted migration of keratinocytes and retained their proliferative capacity.

Neovascularization in wound. Neovascularization was determined at 14 days post-wounding by measuring immunofluorescent staining for CD31 ${ }^{+}$and $\alpha$-smooth muscle actin ${ }^{+}$. The $\mathrm{CD} 1^{+}$positive endothelial cells and $\alpha$-SMA expressing mural cells were evidently observed near the wound margins in the $S$ PLGA + HB-EGF group, as revealed in Fig. 6B, suggesting a new blood vessel formed in the wound region. The HB-EGF + PLGA group formed only a small amount of nascent vessels and other groups did not even form them at all.

\section{Discussion}

Several novel delivery systems showing promising results were developed for the controlled release of growth factors based on sulfated or sulfonated synthetic carbohydrates. ${ }^{17-19}$ Of salient effects are heparin and heparan sulfate proteoglycan, which protect growth factors from proteolytic degradation and increase their bioactivity. What's more, heparin enhanced the ability of some growth factors to bind to their cell receptor. ${ }^{20,21}$ However, heparin stabilized growth factors are water soluble and not able to control release by heparin alone, especially in a cutaneous wound. ${ }^{22}$

In the present study, we designed a heparin-like polysaccharide modified electrospun fiber (S-PLGA) that has a highsurface-area to carry nanoparticles (S-NP) formed by $2-\mathrm{N}, 6-\mathrm{O}$ sulfated chitosan (26SCS) and chitosan. Previous work in our group demonstrated that heparin-like 26SCS had significant effects on BMP-2 and VEGF, even in a low dose of 26SCS. ${ }^{12,13}$ Since HB-EGF has a similar heparin binding domain, we considered both a synergistic effect and controlled release feature might exist between sulfated chitosan and HB-EGF. Thus, the S-PLGA nanofibers incorporated with HB-EGF were fabricated to investigate synergistic effects on cutaneous wounds healing.

Electrospun fibers as high-surface-porous structures provide a superb accommodation for loading HB-EGF. Besides, the sulfated modified nanofibers endowed it with higher affinity and more binding sites for HB-EGF, which improved the loading-efficiency compared to neat PLGA nanofibers. HB-EGF is released from S-PLGA nanofibers along with their degradation. As a result, the existence of S-NP as a heparin-like polysaccharide effectively binds to HB-EGF and decreases the rate of release of HB-EGF, especially a burst release which was reduced from $37 \%$ to $17 \%$ (compared to the neat PLGA nanofibers as a control). It is remarkable that the 26SCS as a nanoscale carrier achieved a controlled and sustainable release of HB-EGF over at least 10 days in vitro with a steady release rate compared with the neat PLGA nanofibers.

Biological evaluation of HB-EGF loaded in vitro was examined by the Scratch and MTS trial with free HB-EGF as a control. The HB-EGF loaded nanofibers greatly potentiated Hacat cells mobility but did not slow their proliferation. Most importantly, our release study showed that HB-EGF existing in the solution of HB-EGF loaded nanofibers was less than $20 \%$ by the end of the $21 \mathrm{~h}$ migration trial; however, the HB-EGF loaded nanofibers induced migration of Hacat cells more effectively than the free HB-EGF ones. Furthermore, the HB-EGF loaded nanofibers showed no obvious difference of cell number compared with the control; in contrast, the free HB-EGF nanofibers suppressed the proliferation of Hacat cells. As a consequence, S-PLGA nanofibers enhanced the bioactivity of HB-EGF and controlled the release of the HB-EGF to avoid inhibiting the proliferation of Hacat cells. Therefore, we hypothesize that 26SCS had a synergistic effect on the migration of Hacat cells with HB-EGF, which affected the wound healing that was further confirmed by the splinted excisional wound model.

The excisional wound splinting model provides an appropriate method for evaluating wound healing in wild-type mice. ${ }^{23}$ Besides, wound closure, granulation, and vasculature can be visualized, measured, and documented at different time points. As demonstrated in Fig. 4, the HB-EGF + S-PLGA group was shown to greatly speed up wound healing compared with other groups. The wound was almost completely closured at 14 days in this group, which healed through re-epithelialization, granulation, and angiogenesis, a process similar to that which occurs in humans.

Endogenous HB-EGF has been shown to play a functional role in re-epithelialization by accelerating keratinocyte migration; however, it was secreted in very low amounts. ${ }^{7,24}$ Thus, application of exogenous HB-EGF is needed for curing a wound. Wound sections were collected to measure cytokeratin, which proved that the HB-EGF + S-PLGA group effectively accelerated re-epithelialization by observing a complete keratinized layer at 7 days after being wounded. Meanwhile, keratinocytes that stained double positive for $\beta 4$ integrin and Ki-67 protein were also observed well beyond the wound margin. ${ }^{25-27}$ This result is consistent with the vitro trial. On the contrary, as to the HB-EGF + PLAG group, we only found the $\beta 4$ integrin that regulated epithelial movement during wound healing was expressed. Proliferation of keratinocyte was inhibited due to the burst release of HB-EGF. Eventually, the rate of wound closure exhibited no significant difference compared with the PLGA nanofibers without HB-EGF as control. Interestingly, the SPLGA nanofibers played favorable roles in proliferation. These results suggested that the 26SCS presumably stimulated keratinocytes around the wound to proliferate, express appropriate integrin receptors, and migrate into the wound space. The mechanism that contributes to these phenomena has yet to be delineated.

After migrating into wounds, fibroblasts begin the synthesis of an extracellular matrix, termed "provisional extracellular," which contributes to the formation of granulation tissue by providing a scaffold. And then the provisional extracellular 
A
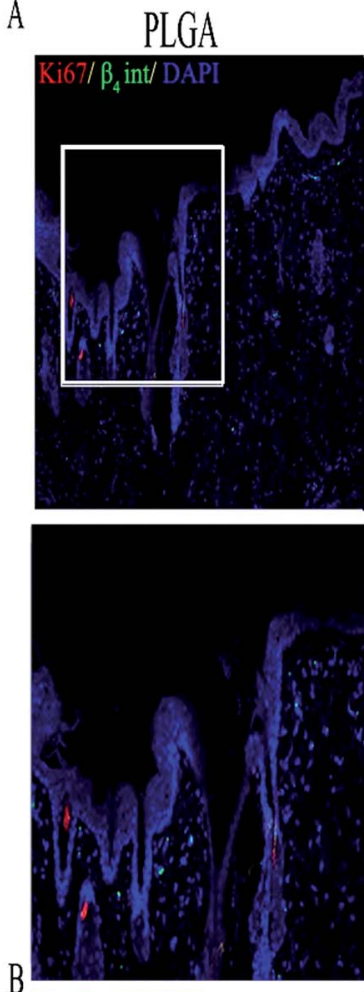

$\mathrm{B}$

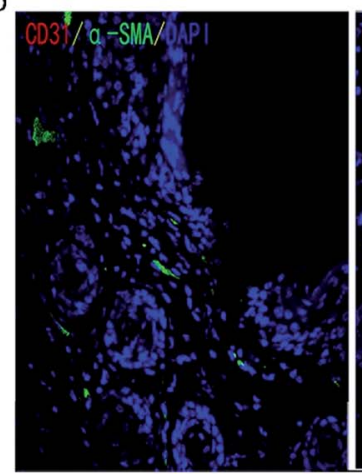

PLGA+HB-EGF
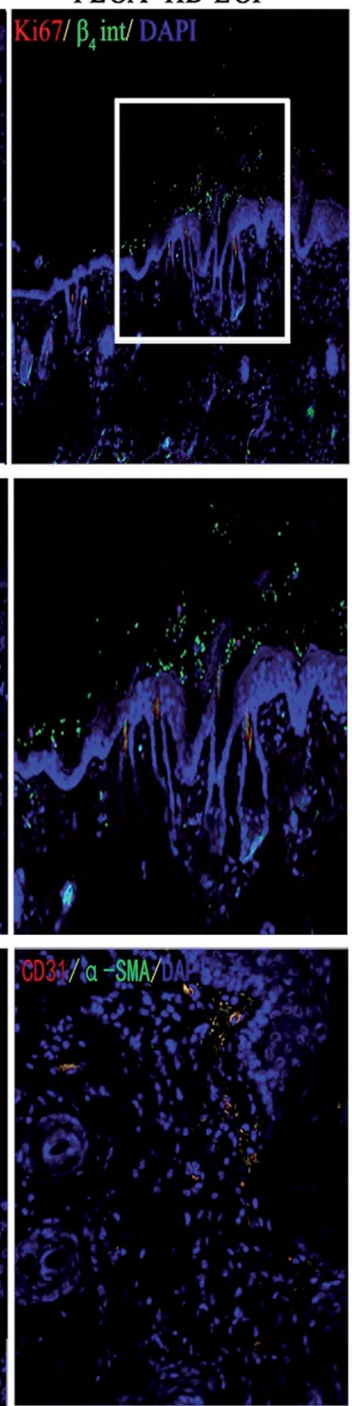
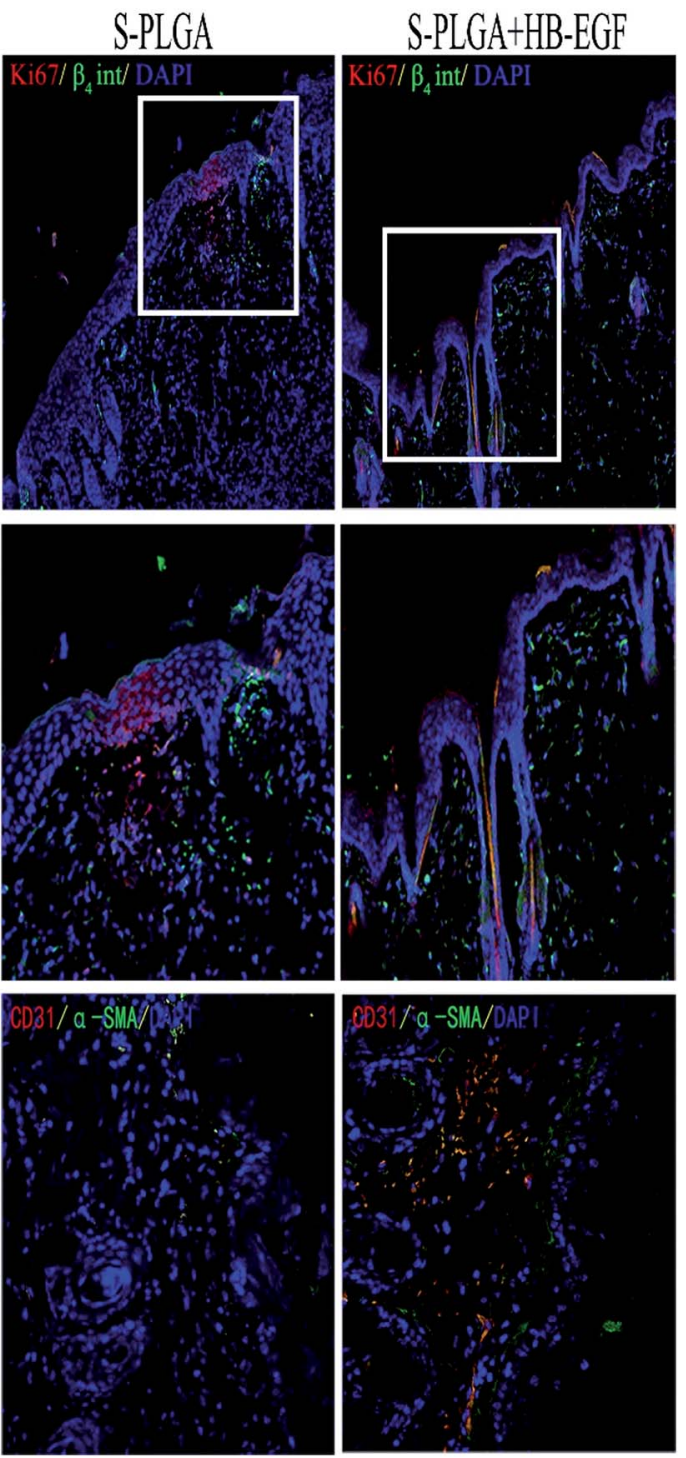

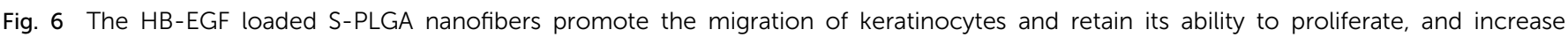

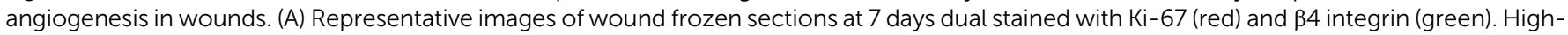

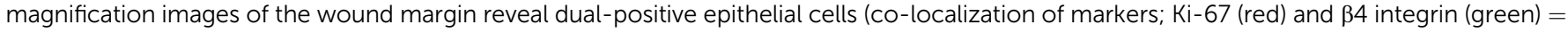
yellow). (B) Representative images of wound frozen sections at 14 days dual stained with CD-31 (red) and $\alpha$-smooth muscle actin (green).

matrix is gradually replaced with a collagenous matrix. The new extracellular matrix is necessary to support cell ingrowth. ${ }^{28,29}$ Some studies described that HB-EGF is mitogenic for fibroblasts and we also observed that high collagen content existed in the HB-EGF + S-PLGA group at 7 days after wounding. ${ }^{\mathbf{8}, 30}$ Besides, the S-PLGA nanofibers without the HB-EGF group and HB-EGF + PLGA group had almost the same amount of collagen content. This possibly resulted from a direct effect of fibroblasts and indirect effect produced by 26SCS as a heparin-like proteoglycan binding to protein ${ }^{31}$ and then altering their orientation in a manner that influences their activity. ${ }^{32}$ Other dermal appendages in the granulation region also were shown in the HB-EGF + S-PLGA group at 14 days after administration. Conversely, the other groups didn't form any appendages, which indicated the HB-EGF-loaded S-PLGA nanofibers realized comprehensive wound healing.
As is known, blood vessels carry oxygen and nutrients to support cell metabolism. Thus, angiogenesis is indispensable to sustain the newly formed granulation tissue during wound healing. However, angiogenesis is a complex process involving the formation of an extracellular matrix in the wound bed as well as migration and mitogenic stimulation of endothelial cells. HB-EGF may affect the process of angiogenesis by initiating paracrine signaling by keratinocytes and fibroblasts in the wound bed. Furthermore, HB-EGF was demonstrated by several studies that it may have a positive effect on endothelial cells and vascular smooth muscle cells. ${ }^{33}$ Besides, the angiogenic process is also regulated by a variety of cytokines. ${ }^{5}$ Particularly important growth factors that contribute to angiogenesis are FGF-2 and VEGF. ${ }^{16}$ The 26SCS as a heparin-like polysaccharide is a necessary cofactor for VEGF and FGF-2. We have assessed that the 26SCS has strong mitogen for angiogenesis with VEGF. ${ }^{\mathbf{1 3 , 1 4}}$ 


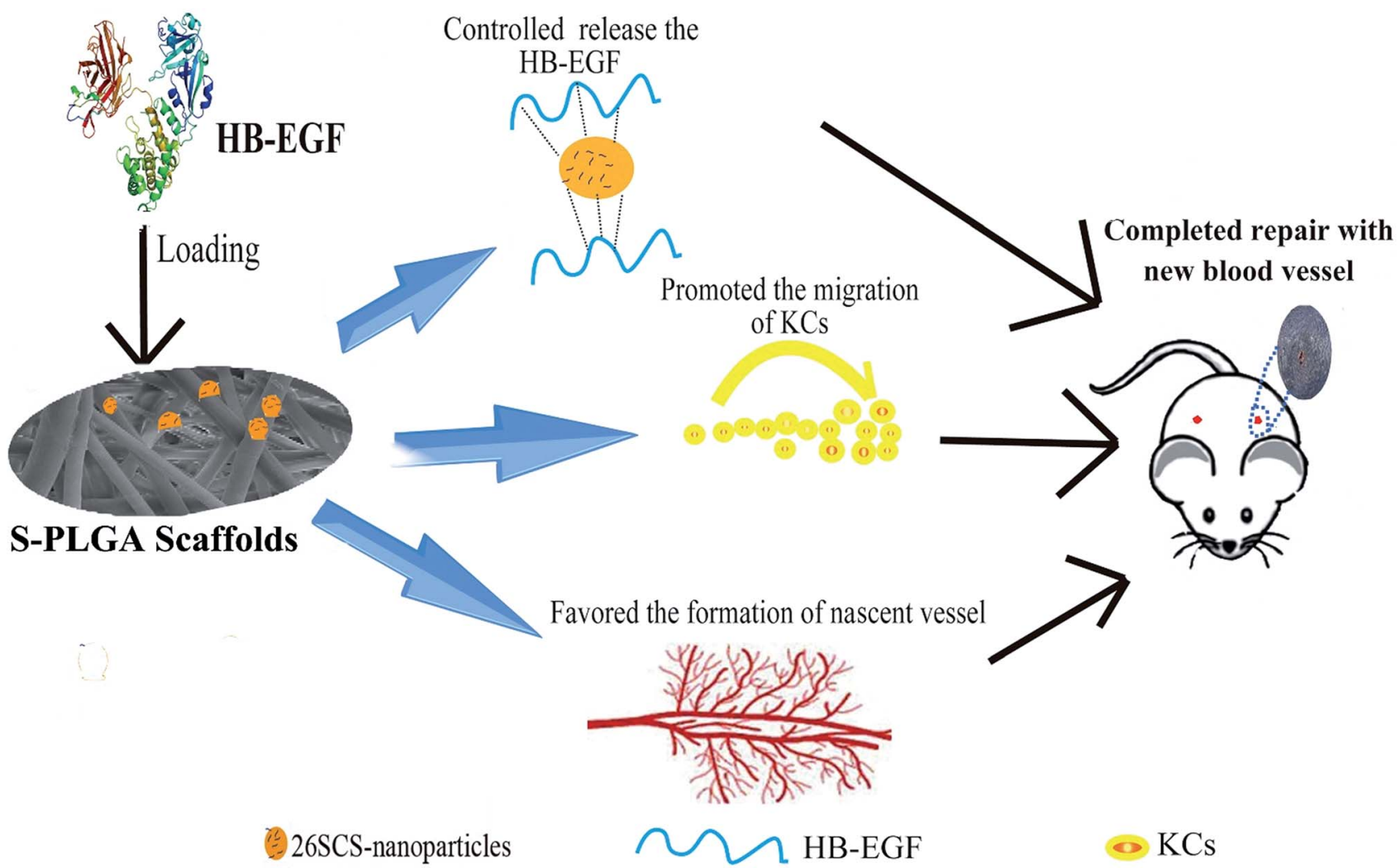

Fig. 7 Schematic diagram of the effect of HB-EGF/S-PLGA on wound healing. (1) The PLGA nanofibers associated with 26SCS sustained and controlled release the HB-EGF, which prolongs its effectiveness. (2) The synergistic effect of 26SCS and HB-EGF promoted migration of the Hacat cells which indicates an enhanced bioactivity of HB-EGF released from the S-PLGA nanofibers. (3) 26SCS may facilitate maturation of the vascular system as a cofactor for VEGF and FGF-2 that contribute to angiogenesis. Combining these, the HB-EGF-loaded S-PLGA nanofibers could potentially complete repairing a wound.

Notably, only the HB-EGF + S-PLGA group showed formation of nascent blood vessels and endothelial cell infiltration. Together with HB-EGF, 26SCS may facilitate maturation of the vascular system in this system, which needs to be further investigated (Fig. 7).

\section{Conclusions}

In this study, PLGA nanofibers were developed to encapsulate sulfated chitosan nanoparticles for HB-EGF loading, which achieved a controlled release of HB-EGF. Furthermore, S-PLGA nanofibers played a significant role in the HB-EGF mediated migration of HACAT cells, without influencing proliferation, which led to acceleration of re-epithelialization. Wounds were almost completely healed at 14 days after application of HBEGF-loaded S-PLGA nanofibers. Besides, 26SCS also had some positive effects on angiogenesis. By this token, these results indicate that HB-EGF-loaded S-PLGA nanofibers can promote comprehensive and fast curing for cutaneous wounds.

\section{Conflicts of interest}

There are no conflicts to declare.

\section{Acknowledgements}

The authors wish to express their gratitude for financial supports from the National Natural Science Foundation of China (No. 31330028, No. 31470923), International Cooperation Project of Shanghai Science and Technique Committee (15520711100), and the Key Project of Logistic Scientific Research of PLA (BWS13C014). This study was also supported by the 111 Project (B14018).

\section{Notes and references}

1 M. G. Franz, M. C. Robson, D. L. Steed, A. Barbul, H. Brem, D. M. Cooper, D. Leaper, S. M. Milner, W. G. Payne, T. L. Wachtel and L. Wiersema-Bryant, Wound Repair Regen., 2008, 16, 723-748.

2 Y. Yamaguchi and K. Yoshikawa, J. Dermatol., 2001, 28, 521534.

3 S. F. Badylak, D. O. Freytes and T. W. Gilbert, Acta Biomater., 2009, 5, 1-13.

4 S. Guo and L. A. DiPietro, J. Dent. Res., 2010, 89, 219-229.

5 B. Behm, P. Babilas, M. Landthaler and S. Schreml, J. Eur. Acad. Dermatol. Venereol., 2012, 26, 812-820. 
6 M. Marikovsky, K. Breuing, P. Y. Liu, E. Eriksson, S. Higashiyama, P. Farber, J. Abraham and M. Klagsbrun, Proc. Natl. Acad. Sci. U. S. A., 1993, 90, 3889-3893.

7 Y. Shirakata, R. Kimura, D. Nanba, R. Iwamoto, S. Tokumaru, C. Morimoto, K. Yokota, M. Nakamura, K. Sayama, E. Mekada, S. Higashiyama and K. Hashimoto, J. Cell Sci., 2005, 118, 2363-2370.

8 R. K. Cribbs, P. A. Harding, M. H. Luquette and G. E. Besner, J. Burn. Care Res., 2002, 23, 116-125.

9 J. S. Choi, K. W. Leong and H. S. Yoo, Biomaterials, 2008, 29, 587-596.

10 E. R. Edelman, M. A. Nugent, L. T. Smith and M. J. Karnovsky, J. Clin. Invest., 1992, 89, 465-473.

11 S. E. Sakiyama-Elbert and J. A. Hubbell, J. Controlled Release, 2000, 65, 389-402.

12 L. Cao, J. A. Werkmeister, J. Wang, V. Glattauer, K. M. McLean and C. Liu, Biomaterials, 2014, 35, 2730-2742.

13 Y. Yu, J. Chen, R. Chen, L. Cao, W. Tang, D. Lin, J. Wang and C. Liu, ACS Appl. Mater. Interfaces, 2015, 7, 9982-9990.

14 L. Cao, J. Wang, J. Hou, W. Xing and C. Liu, Biomaterials, 2014, 35, 684-698.

15 A. J. Melchiorri, N. Hibino, T. Yi, Y. U. Lee, T. Sugiura, S. Tara, T. Shinoka, C. Breuer and J. P. Fisher, Biomacromolecules, 2015, 16, 437-446.

16 A. J. Singer and R. A. F. Clark, N. Engl. J. Med., 1999, 341, 738746.

17 H. Chu, N. R. Johnson, N. S. Mason and Y. Wang, J. Controlled Release, 2011, 150, 157-163.

18 O. Jeon, C. Powell, L. D. Solorio, M. D. Krebs and E. Alsberg, J. Controlled Release, 2011, 154, 258-266.

19 F. Zomer Volpato, J. Almodovar, K. Erickson, K. C. Popat, C. Migliaresi and M. J. Kipper, Acta Biomater., 2012, 8, 1551-1559.
20 D. H. Damon, R. R. Lobb, P. A. D'Amore and J. A. Wagner, J. Cell. Physiol., 1989, 138, 221-226.

21 T. Spivak-Kroizman, M. A. Lemmon, I. Dikic, J. E. Ladbury, D. Pinchasi, J. Huang, M. Jaye, G. Crumley, J. Schlessinger and I. Lax, Cell, 1994, 79, 1015-1024.

22 O. Saksela, D. Moscatelli, A. Sommer and D. B. Rifkin, J. Cell Biol., 1988, 107, 743-751.

23 X. Wang, J. Ge, E. E. Tredget and Y. Wu, Nat. Protoc., 2013, 8, 302-309.

24 S. W. Stoll, L. Rittié, J. L. Johnson and J. T. Elder, J. Invest. Dermatol., 2012, 132, 2148-2157.

25 S. N. Nikolopoulos, P. Blaikie, T. Yoshioka, W. Guo, C. Puri, C. Tacchetti and F. G. Giancotti, Mol. Cell. Biol., 2005, 25, 6090-6102.

26 A. J. Russell, E. F. Fincher, L. Millman, R. Smith, V. Vela, E. A. Waterman, C. N. Dey, S. Guide, V. M. Weaver and M. P. Marinkovich, J. Cell Sci., 2003, 116, 3543-3556.

27 T. Scholzen and J. Gerdes, J. Cell. Physiol., 2000, 182, 311322.

28 G. C. Gurtner, S. Werner, Y. Barrandon and M. T. Longaker, Nature, 2008, 453, 314-321.

29 J. L. Monaco and W. T. Lawrence, Clin. Plast. Surg., 2003, 30, 1.

30 A. Rosenthal, P. B. Lindquist, T. S. Bringman, D. V. Goeddel and R. Derynck, Cell, 1986, 46, 301-309.

31 S. Werner, T. Krieg and H. Smola, J. Invest. Dermatol., 2007, 127, 998-1008.

32 L. Pellegrini, D. F. Burke and F. V. Delft, Nature, 2000, 407, 1029-1034.

33 V. B. Mehta, Y. Zhou, A. Radulescu and G. E. Besner, Growth Factors, 2008, 26, 301-315. 